\title{
Experimental investigation of the atmospheric steam engine with forced expansion
}

Authors: Gerald Müller, Senior Lecturer, Faculty of Engineering and the Environment, University of Southampton, Southampton SO17 1BJ, UK. Tel.: +44 2380 592465, email: g.muller@soton.ac.uk

George Parker, Undergraduate Student, Faculty of Engineering and the Environment, University of Southampton, Southampton SO17 1BJ,

Abstract: Low and medium temperature thermal energy with temperatures of $100^{\circ}$ to $150^{\circ} \mathrm{C}$ is available from renewable energy sources such as solar thermal or geothermal energy. Recent progress in flat plate solar thermal collector technology indicates that economical solutions for this temperature range are now becoming possible. Current technologies to generate mechanical energy from this temperature bracket such as Organic Rankin Cycle machines are however complex, and therefore only economical for larger units. There is a need for a simple, cost-effective medium temperature thermal engine for small scale applications. Recently, the atmospheric steam engine was re-evaluated for this application. The theory was extended to include a forced expansion strike. This can increase the theoretical efficiency of the ideal engine from $6.5 \%$ to $20 \%$. In order to assess this theory, a series of experiments was conducted at Southampton University. It was found that the isothermal expansion of steam, and its subsequent condensation, is possible. The experiments showed a maximum efficiency of $10.2 \%$ for an expansion ratio of $1: 4$, indicating the validity of the theory. A further increase of efficiency to approximately $17 \%$ appears possible. It was concluded that the atmospheric engine with forced expansion has development potential.

\section{Keywords:}

Thermal engine, low and medium temperature, solar thermal energy, steam engine

\section{Introduction}

\subsection{Overview}

Low and medium temperature thermal energy is generated in many areas of renewable energy, such as biomass or solar energy, as well as in industrial processes. Solar thermal energy here probably constitutes the most abundant resource, which is also expected to 
grow in many areas of Europe with the effects of climate change becoming more pronounced [1]. Currently it is mostly employed for domestic heating (low temperature, < $80^{\circ} \mathrm{C}$ ), and for energy generation at large scale installations (high temperature, often > $\left.400^{\circ} \mathrm{C}\right)$. Cost-effective, medium temperature $\left(100-200^{\circ} \mathrm{C}\right)$, medium scale systems e.g. for applications in industry or commercial companies however still require development. The availability of a simple, efficient and economical thermal engine for this temperature range and for power ratings between 5 and $100 \mathrm{~kW}$ would widen the potential area of application of solar thermal energy significantly. Ongoing research at Southampton University aims at the development of a cost-effective solar thermal system for low- and medium temperatures of $100^{\circ}$ to $180^{\circ} \mathrm{C}$. The system comprises a collector, and a thermal engine to generate mechanical from thermal energy. In this article, recent developments of the thermal engine are described.

\subsection{Solar thermal energy}

There is a large variety of technologies available for the harvesting of solar thermal energy available, see e.g. the overview in [2] or [3]. Flat plate or non-concentrating solar thermal collectors are probably the most economical collector types for solar thermal energy. Their main disadvantage is the comparatively low operating temperature (usually below $80^{\circ} \mathrm{C}$ ), which makes them not suitable for many processes such as power generation.

Commercially available collectors are mostly designed for operating temperatures below $100{ }^{\circ} \mathrm{C}$, high performance collectors can reach this temperature with $37 \%$ efficiency (assuming a solar energy of $G=800 \mathrm{~W} / \mathrm{m}^{2} \mathrm{~K}$ ), e.g. [4].

Recent developments of higher efficiency flat-plate, solar thermal collectors for low and medium temperatures of 120 to $200 \mathrm{C}$ are however promising and may have the potential to open up this field of solar energy:

ISFH/Germany developed a double glazed flat plate collector with an Argon-filled cavity between glass plates, low-e glass, absorbing paint and with increased insulation [5]. From their data, an efficiency of $24 \%$ could be calculated for a temperature difference of $126 \mathrm{~K}$ with a solar radiation of $G=800 \mathrm{~W} / \mathrm{m}^{2} \mathrm{~K}$. This would correspond to an operating temperature of $144^{\circ} \mathrm{C}$, assuming an ambient temperature of $20^{\circ} \mathrm{C}$. Losses amounted to $3.5 \mathrm{~W} / \mathrm{m}^{2} \mathrm{~K}$, indicating the potential of flat plate collectors.

Recent development work at Southampton University focussed on a low-cost solar thermal collector built from standard building materials and low-iron glass. The collector employed a large air gap of $150 \mathrm{~mm}$, double glazing and passive convection control. Losses were 
measured as $2.3 \mathrm{~W} / \mathrm{m}^{2} \mathrm{~K}$ at $\Delta T=126 \mathrm{~K}$ [6]. With a solar radiation intensity of $G=800 \mathrm{~W} / \mathrm{m}^{2}$, the collector could reach an operating temperature of $144^{\circ} \mathrm{C}$ with an efficiency of $45 \%$.

The development of a cost-effective and efficient solar thermal energy supply therefore has reached a stage where the next step, the development of a low temperature thermal engine for decentralised small-scale application, is required.

\subsection{Thermal machines for low and medium temperatures}

Several technologies for the conversion of thermal energy in this temperature range into mechanical and electrical energy exist. The most common principles for energy conversion are hot air engines (Stirling engines), and Organic Rankin Cycle (ORC) engines. Hot air engines employ the expansion of air when heated, and contraction when cooled. Their conversion efficiency for medium temperature situations is however quite low. Tests with a low temperature Stirling engine resulted in an efficiency of $0.44 \%$ for a heater temperature $T_{\mathrm{Ev}}$ of $166^{\circ} \mathrm{C}$, [7]. ORC engines utilise working fluids with evaporation temperatures well below $100^{\circ} \mathrm{C}$. The fluid is evaporated under pressures of 6 to 20 bar with temperatures of 80 to $180^{\circ} \mathrm{C}$. The steam drives a turbine, and is then condensed to be evaporated again. Theoretical efficiencies are a function of the boiler temperature and the type of fluid used. Simulations for different fluids and evaporation temperatures gave efficiencies of $5.6 \%$ for $T_{\mathrm{Ev}}=86^{\circ} \mathrm{C}, 7.7 \%$ for $T_{\mathrm{Ev}}=109^{\circ} \mathrm{C}$, and $13.1 \%$ for $T_{\mathrm{Ev}}=169^{\circ} \mathrm{C}$, [8]. In experimental investigations, an efficiency of $7.98 \%$ was reported for an operating temperature of $120^{\circ} \mathrm{C}$ and a pressure of 9 bar [9]. The system is however quite complex and comprises an evaporator, turbine, scroll condenser, pumps and a regenerator. This complexity, combined with the design requirements for a pressurized, expensive fluid means that smaller units $(\mathrm{P}<$ $150 \mathrm{~kW}$ ) are difficult to produce cost-effectively. Today, ORC thermal machines are mostly used in the fields of biomass and geothermal energy, and waste heat recovery. A promising area for application is seen in small scale solar thermal systems with Fresnel concentrators which deliver lower temperatures than e.g. parabolic trough systems, but require lower investment costs [10].

\section{The atmospheric steam engine}

\subsection{Historical development}

The atmospheric steam engine is the oldest type of practical steam engine. It was initially developed by Thomas Newcomen in 1712, and significantly improved by James Watt with the introduction of the external condenser in 1776. The ASE operates at atmospheric pressure, and employs a vacuum generated by the condensation of steam as driving force. 
In the simplest version, the machine consists of a boiler, a cylinder with an inlet for cold water, and a piston, Fig. 1a. During the upwards motion, steam is drawn into the cylinder. When the uppermost position is reached, the boiler valve is close and cold water injected into the cylinder. The steam condenses, a near vacuum forms and the atmospheric pressure drives the piston downwards.

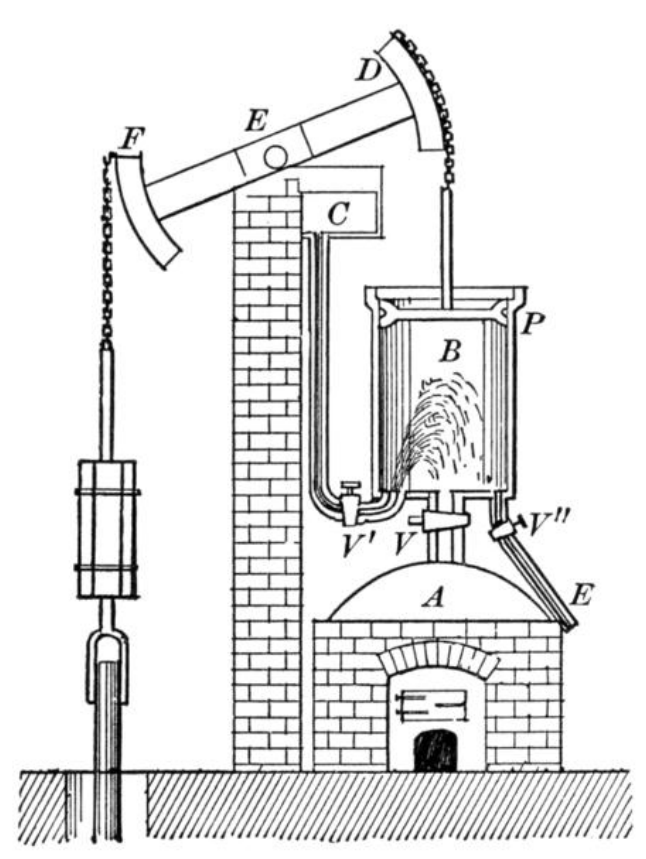

a. Newcomen's atmospheric engine [11]

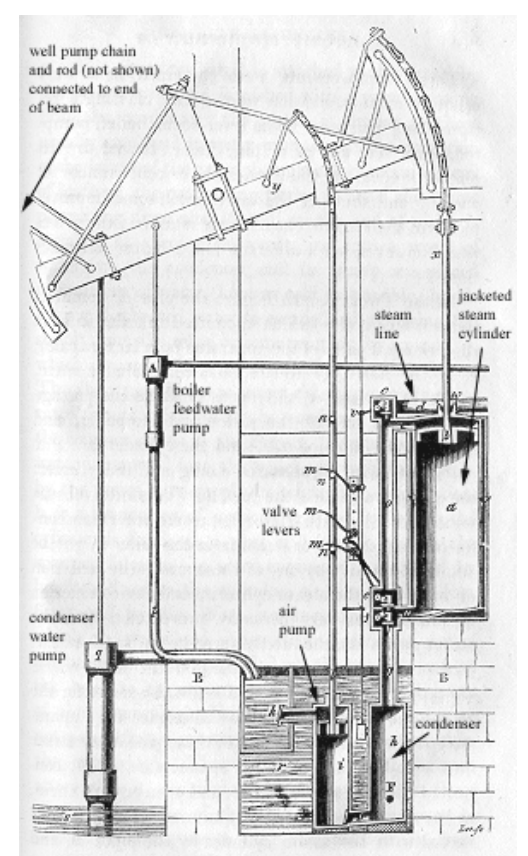

b. Watt's engine with external condenser [12]

Fig. 1: Historic machines

Newcomen's engine had very low efficiencies of approximately $0.5 \%$, [13], since with every injection of cold water the cylinder cooled down, and steam had to be employed to heat it up again. James Watt introduced the external condenser in 1776. An additional small vacuum cylinder was added to the machine, Fig. 1b. During the upward motion of the piston, steam was drawn into the main cylinder. In the condenser, the piston was also moved upwards to create a near vacuum. When the working piston reached the uppermost position, the boiler valve was closed and the condenser valve opened. The vacuum drew steam into the condenser where it condensed into water, maintaining the vacuum and drawing more steam. This machine had the great advantage that the working piston remained hot, and the condenser cold so that efficiencies were increased to $3.5 \%$, [13].

The theory shows that the atmospheric engine can only recover the displacement work of the water as it evaporates and displaces $1.69 \mathrm{~m}^{3}$ of atmosphere for $1 \mathrm{~kg}$ (or $0.001 \mathrm{~m}^{3}$ ) of 
water. This work is, in the ideal case, $169 \mathrm{~kJ} / \mathrm{kg}$. In the same time, the thermal energy required to heat $1 \mathrm{~kg}$ of water to $100^{\circ} \mathrm{C}$, and to evaporate it, amounts to $2601.5 \mathrm{~kJ} / \mathrm{kg}$, so that the maximum theoretical efficiency is only $6.5 \%$.

With the advent of high pressure machines, the atmospheric engine disappeared. The main reason was the limited efficiency of the atmospheric engine. The atmospheric engine does however have several advantages:

1. Simplicity,

2. It uses a cheap. non-toxic, not inflammable working fluid,

3. It operates at very low temperatures compared with other thermal engines,

4. It operates under atmospheric or sub-atmospheric pressures so that there is no danger of boiler explosions. This reduces manufacturing and maintenance / certification costs substantially.

The last application of the atmospheric steam engine known to the author is the machine designed by Davey in 1884, [14]. Davey advocated the design on the grounds mentioned above plus the fact that, since there is no danger of boiler explosions, the machine could be situated anywhere, even in residential areas.

\subsection{Recent developments}

Recently, the concept of the atmospheric engine was revisited in order to assess its potential for the utilisation of low temperature thermal energy, [15]. The classic atmospheric cycle described in the previous section was modified to include a forced expansion of the steam. The theoretical work indicated that the efficiency of the atmospheric steam engine could be increased from $6.5 \%$ to $20 \%$.

In a forced expansion cycle, initially a certain volume of steam is drawn into the cylinder. The boiler valve is closed. The piston is then drawn upwards in order to expand the steam. The mechanical work required for the expansion is the integral of the external force applied over the expansion length. This force is zero at the beginning of the expansion, and reaches a maximum at the end of the expansion. The maximum expansion force is therefore always significantly smaller than the atmospheric force acting on the piston from the outside. Once the prescribed expansion ratio is reached, condensation is initiated. The atmospheric force now conducts work over the full length of the stroke (initial steam volume plus expansion length).

In [15], the theory of the ASE with forced expansion was presented for an adiabatic expansion of the steam. In a real engine however, the cylinder will remain hot so that the expansion there will be isothermal: For an initial volume $v_{1}$, and a given expansion ratio $n=$ 
$162 v_{2} / v_{1}$, the expanded pressure is $p_{2}=p_{1} / n$. For a cylinder cross sectional area of $A_{C y l}=1 \mathrm{~m}^{2}$,

163 the expansion work $W_{\text {exp }}$ becomes:

$164 \quad W_{\text {exp }}=p_{1} \cdot\left(v_{2}-v_{1}\right)-p_{1} \cdot v_{1} \cdot \ln \frac{p_{2}}{p_{1}}$

Where $p_{1}=p_{\text {atm }}$.

166 The total work of the system $W_{\text {tot }}$ after condensation of the steam is:

$167 W_{t o t}=p_{a t m} \cdot v_{2}-W_{\text {exp }}$

168 The thermal energy $W_{\text {isoth }}$ which has to be supplied to the expanded steam in order to maintain its temperature is small, due to the low adiabatic coefficient $\mathrm{K}$ of wet steam $(\mathrm{K}=$ 1.035 to $1.08,[16])$. The thermal energy required ranges from $0(n=1)$ to $5 \%(n=12)$ of the isothermal expansion work $W_{\text {exp }}$, and can be calculated from the temperature drop in the adiabatic expansion. It is included in Fig. 2. The thermal energy input $E_{\mathrm{th}}$ required for a given volume of steam $v_{1}=1 \mathrm{~m}^{3}$ can be calculated as follows (all units in $\mathrm{m}, \mathrm{J}, \mathrm{K}$ and $\mathrm{kg}$ ):

$E_{t h}=(2,256,500+4,200 \cdot 70) \cdot \frac{v_{1}}{1.69}+W_{\text {isoth }}$

$2,256.5 \mathrm{~kJ} / \mathrm{kg}$ is the latent heat of water, the specific heat capacity of water is $4,200 \mathrm{~J} / \mathrm{kgK}$, an initial temperature of $30^{\circ} \mathrm{C}$ is assumed for the water and $1 \mathrm{~kg}$ of water amounts to $1.69 \mathrm{~m}^{3}$ of steam. For the calculation of the thermal efficiency it is assumed that the expansion work is provided by the work generated by the machine, and therefore has to be subtracted from the condensation work. The thermal efficiency $\eta$ then becomes:

$180 \quad \eta=\frac{W_{t o t}}{E_{t h}}$

Fig. 2 shows the theoretical efficiency from a forced expansion stroke as a function of the expansion ratio $n$ for both adiabatic and isothermal expansion. The efficiency ranges from

1830.065 for $n=1$ to 0.198 for $n=10$. Isothermal expansion gives in marginally higher efficiencies, and approximately $3 \%$ more power output per unit volume compared with adiabatic expansion.

186 It should be noted that the Carnot efficiency limit does not apply directly here since there is 187 an additional energy input - the expansion force - into the system. A more detailed 188 discussion of this aspect is given in [15]. 


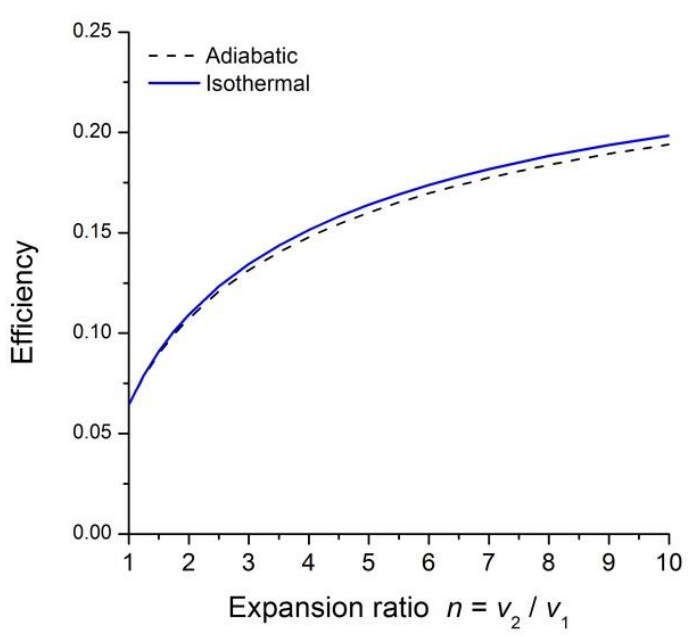

189

190 Fig. 2: Theoretical efficiency as function of the expansion ratio $n$.

191 Forced expansion appears to open the possibility to create a thermal engine for temperatures of $100^{\circ} \mathrm{C}$ with efficiencies exceeding those from ORC engines, whilst avoiding complex pressurized systems with expensive working fluids.

\section{Experiments}

\subsection{Experimental set-up}

The experiment was designed in order to assess the theory of isothermal forced expansion. The specific aims were:

1. To establish whether or not the isothermal expansion of steam is possible, and

2. To determine the efficiencies of a working stroke without and with forced expansion. In order to reach these two aims, a simple one-stroke bench model was designed. It consists of a vertical cylinder, a piston, a boiler, a condenser and a load rig which allows to lift the piston. The forces acting on the cylinder are measured with a scale attached to the lifting rope. The piston movement was controlled with a winch.

Fig, 3a shows the cylinder itself. It consists of a brass base plate $220 \times 220 \mathrm{~mm}, \mathrm{t}=10 \mathrm{~mm}$, a brass inner cylinder of $400 \mathrm{~mm}$ height with an outer diameter of 56 and an inner diameter of $47 \mathrm{~mm}$, and an outer cylinder of $100 \mathrm{~mm}$ inner diameter. The space between outer and inner cylinder is filled with boiling water in order to maintain the working temperature inside. Additional insulation material (polyurethane foam) of approximately $50 \mathrm{~mm}$ thickness was added round the cylinder to prevent further heat losses. The piston was made of stainless steel, with a diameter of $46.5 \mathrm{~mm}$, a length of $75 \mathrm{~mm}$ and a mass of $0.95 \mathrm{~kg}$. Two O-rings were used to seal the piston. The cylinder was mounted on a frame made from aluminium profiles. The copper pipes and taps / switches required for operation were fixed to a wooden 

system.

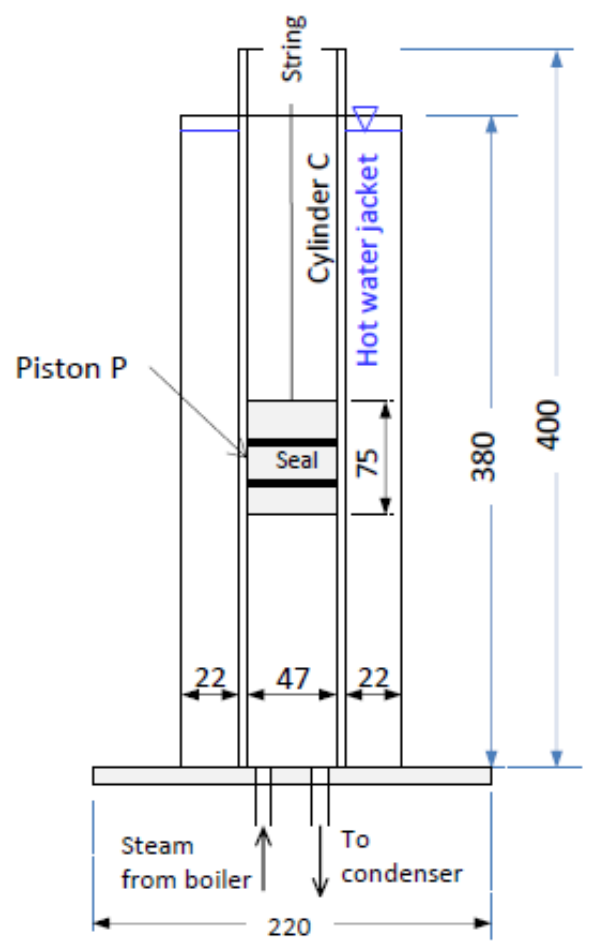

216

217
a. Cylinder and piston

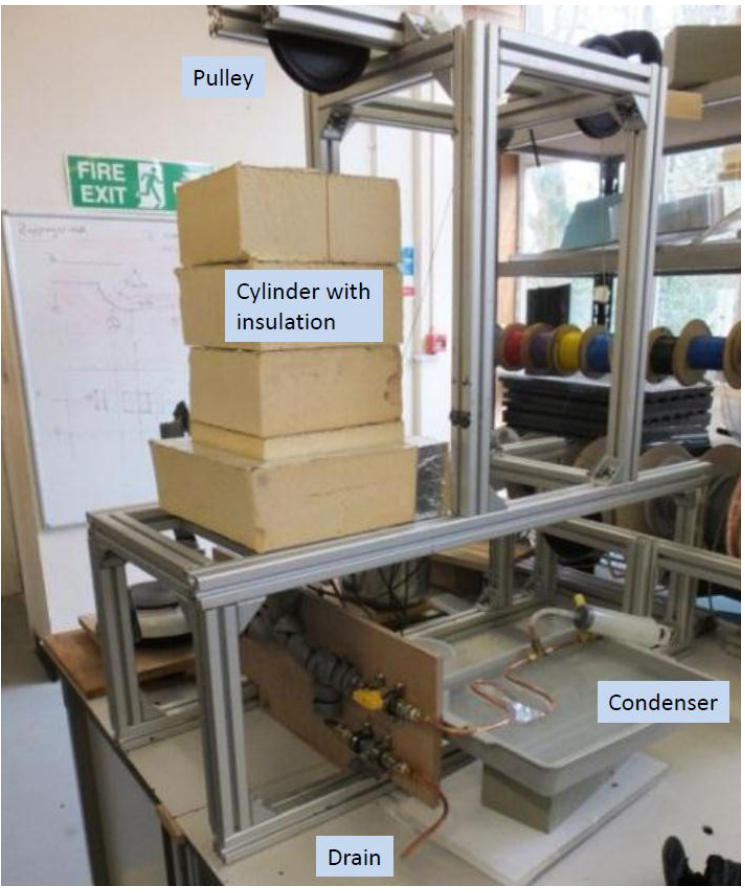

b. Complete apparatus

218 Fig. 3: Experimental set-up

219 A $205 \mathrm{~mm}$ diameter kettle with a maximum content of $5 \mathrm{l}$ was used as boiler. During the 220 experiments it was found that a volume of 0.825 I was evaporated within 30 minutes, 221 corresponding to a delivery of $0.78 \mathrm{I}$ of steam per second. 


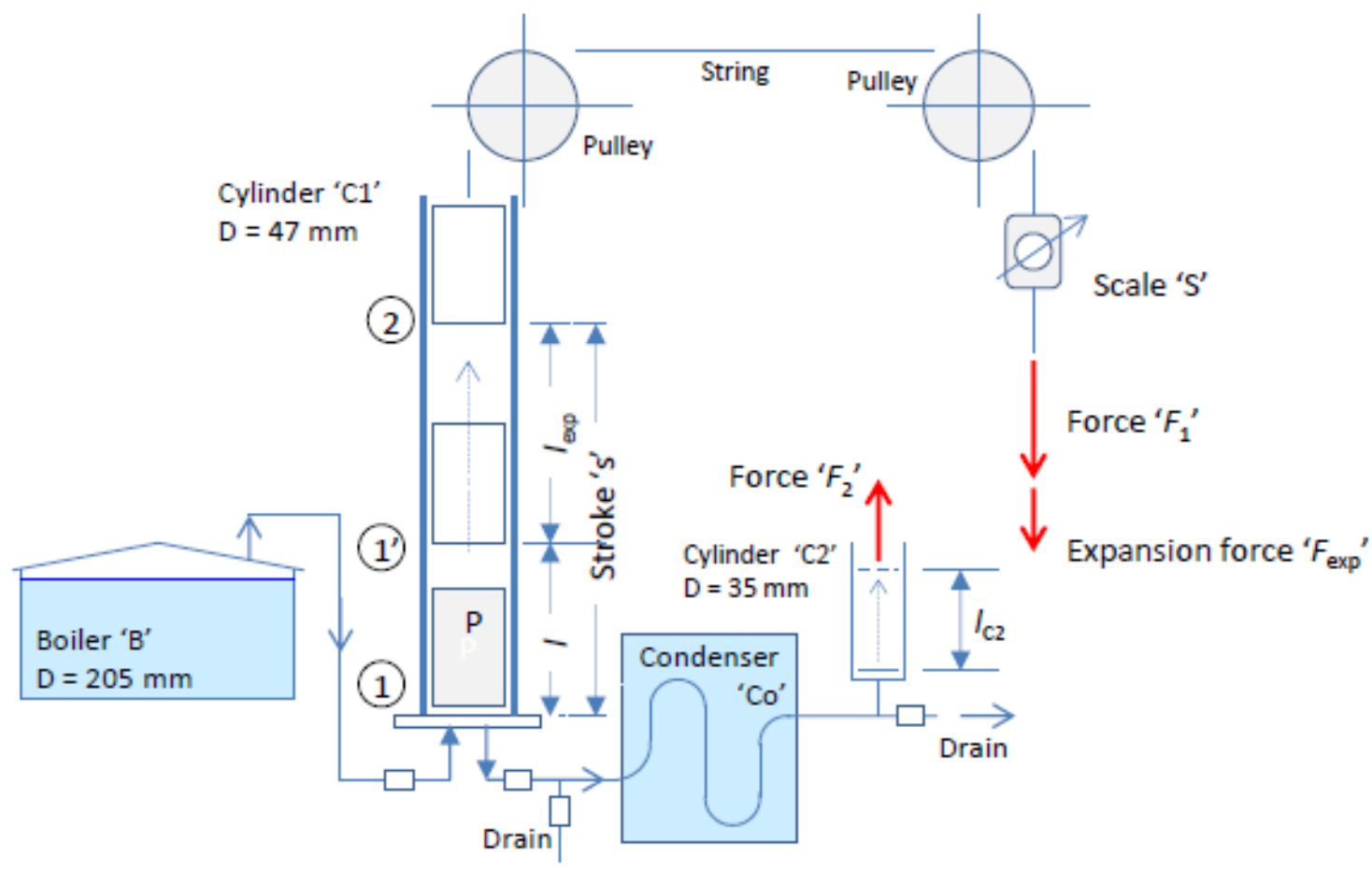

223 Fig. 4: System with external forces

224 The condenser was built from an $800 \mathrm{~mm}$ long copper tube with an external diameter of 6

$225 \mathrm{~mm}$, and an internal diameter of $3 \mathrm{~mm}$. The condenser is connected to the working cylinder

226 C1 with a tap which allows to open or close the connection. A drain tap is also attached so

227 that after every stroke the condenser can be cleared of condensation water. In the

228 condensation section, the copper tube was bent into a W-shape which in turn was set into a

229 basin with cold water. A $100 \mathrm{ml}$ medical syringe with an internal diameter of $35 \mathrm{~mm}$ was

230 used as cylinder $\mathrm{C} 2$ to create a low pressure inside the condenser initially, and then to

231 evacuate the air which leaked into the cylinder $\mathrm{C} 1$ during the working stroke. The boiler $\mathrm{B}$ is

232 connected to the working cylinder $\mathrm{C} 1$. Inside $\mathrm{C} 1$ runs the piston $\mathrm{P}$. attached to $\mathrm{P}$ is a string

233 which runs over two pulleys and is connected to a scale. The external load is applied at this

234 point.

3.2 Tests

\subsubsection{Overview}

Two series of tests were conducted:

(1) Series 1 with condensation only,

(2) and series 2 with forced expansion.

241 The working stroke length $s$ was constant for all tests with $s=200 \mathrm{~mm}$. Initial steam volumes varied with $I=50$ to $200 \mathrm{~mm}$, and expansion lengths of $I_{\exp }=50$ to $150 \mathrm{~mm}$. Before the 
273 tests, the rig was heated up by filling the jacket with boiling water, and by drawing and expelling steam several times. The condensation which formed initially was thereby drained as well. For every expansion ratio, a four strokes were measured. Friction forces were determined as $28 \mathrm{~N}$ upwards, and $6 \mathrm{~N}$ downwards. These forces were added to (downwards) or subtracted from (upwards) the force measurements in order to obtain the actual forces acting on the piston.

\subsubsection{Series 1: condensation only}

The piston $P$ is lifted from the starting position at point ' 1 ' by a distance ' $s$ ', filling $C 1$ with steam. When point ' 2 ' is reached, the boiler valve is closed, and a force is applied to the cylinder $\mathrm{C} 2$ to create a low pressure in the condenser $\mathrm{Co}$. Then the condenser valve is opened, condensation occurs, the pressure in the cylinder drops, and the piston $P$, which is initially held in position, is released slowly back to position ' 1 '. The force $F_{1}$ acting on $\mathrm{P}$ is measured with scale $S$ at the beginning $\left(F_{12}\right.$. pos. 2$)$ and the end of the working stroke $\left(F_{11}\right.$, pos. 1). While the piston $P$ moves, the plunger in Cylinder $\mathrm{C} 2$ is lifted through a distance $I_{\mathrm{C} 2}$, which was constant in this test series at $I_{\mathrm{C} 2}=80 \mathrm{~mm}$, by applying a force $F_{2}$ in order to extract the air from $\mathrm{C} 1$, and thus to allow $\mathrm{P}$ to return to Pos. 1. The work is then calculated as follows:

Condensation work $W_{\text {cond: }}$ :

$W_{\text {cond }}=F_{1} \cdot s=\frac{F_{11}+F_{12}}{2} \cdot s$

The work $W_{\mathrm{C} 2}$ conducted at $\mathrm{C} 2$ is calculated by reducing the force $F_{1}$ with the area ratio of cylinders $\mathrm{C} 1$ and $\mathrm{C} 2$ (assuming that the pressure in the system is the same everywhere):

$W_{C 2}=F_{1} \cdot \frac{D_{2}^{2}}{D_{1}^{2}} \cdot l_{C 2}$

Total work $W_{\text {tot: }}$

$W_{\text {tot }}=W_{\text {cond }}-W_{C 2}$

The tests showed that the seal was not perfect, and therefore a complete vacuum could not be achieved. Cylinder pressures at condensation only reached an average value of $p_{\text {cond }}=$ $46 \mathrm{kPa}$ (abs.). The maximum theoretical efficiency of an atmospheric cycle with a perfect vacuum (i.e. a pressure difference of $100 \mathrm{kPa}$ ) is $6.5 \%$. With a residual pressure $46 \mathrm{kPa}$ (abs.), the maximum theoretical efficiency $\eta_{\text {theor }}$, becomes

$\eta_{\text {theor }}=\frac{100-46}{100} \cdot 6.5=3.5 \%$ 
274 In addition, thermal energy is required to heat the air drawn first into $\mathrm{C} 1$, and then into $\mathrm{C} 2$.

275 With a specific heat capacity of air of $717 \mathrm{~J} / \mathrm{kgK}$ and a density of air at atmospheric pressure

276 of $\rho_{\text {air }}=1.25 \mathrm{~kg} / \mathrm{m}^{3}$ the energy $E_{\text {Air }}$ required becomes:

$277 \quad E_{\text {air }}=717 \cdot 1.25 \cdot \frac{0.035^{2}}{4} \cdot \pi \cdot l_{C 2} \cdot \frac{p_{\text {atm }}-p_{\text {cond }}}{p_{\text {atm }}}$

278 With a specific heat capacity of the water of $4.2 \mathrm{~kJ} / \mathrm{kgK}$, the required thermal energy $E_{\text {th }}$

279 (assuming an initial temperature of the water of $30^{\circ} \mathrm{C}$ ) then is:

$280 \quad E_{t h}=A_{C 1} \cdot l \cdot(70 \cdot 4200+2256,500) / 1.69+E_{\text {air }}$

281 The total energy $W_{\text {tot }}$ becomes

$282 \quad W_{t o t}=W_{c o n d}-W_{C 2}$

283 With this input energy, the efficiency $\eta$ can be calculated:

$284 \quad \eta=\frac{W_{t o t}}{E_{t h}}$

285

286

3.2.3 Series 2 (with forced expansion):

287

Steam is drawn in from the boiler for the initial length ' $"$ from pos. 1 to 1 '. Then the boiler valve is closed. The piston is lifted further through the expansion stroke length 'lexp' to pos. 2 by applying a force $F_{\text {exp }}$, which varies from zero (pos. 1') to a maximum value at pos. 2. A force is applied at the cylinder C2 to create a low pressure in the condenser Co. Then, the condenser valve is opened. Condensation takes place, resulting in a sudden increase in $F_{12}$. The piston $\mathrm{P}$ is still held in position ' 2 '. A force $F_{2}$ is applied at $\mathrm{C} 2$ to draw the air from cylinder $\mathrm{C} 1$ in until the piston $\mathrm{P}$ reaches pos. 1 again, with a reduced end force $F_{11}$ acting now on the piston. The work is then calculated as follows:

Condensation work $W_{\text {cond }}$ :

296

$W_{\text {cond }}=F_{1} \cdot s=\frac{F_{11}+F_{12}}{2} \cdot s$

297

The expansion pressures measured in test series 1 did not correspond well with the theoretical values for the measured expansion lengths. This led to the conclusion that air was drawn into $\mathrm{C} 1$ during the expansion stroke. Using the theory of isothermal expansion in order to calculate the expansion work was considered as inadequate. In order to determine the expansion work, a linear variation from zero to $F_{\text {exp }}$ was therefore assumed. 
$303 \quad W_{\exp }=\frac{F_{\text {exp }}}{2} \cdot l_{\exp }$

304

305 Work in cylinder C2:

306 With condensation, a force $F_{2}$ acts on the plunger in C2. The plunger is moved upwards by a

307 distance $I_{\mathrm{C} 2}$, which varied from 80 to $94 \mathrm{~mm}$, in order to remove air from $\mathrm{C} 1$. Work conducted 308 at $\mathrm{C} 2$ :

$309 \quad W_{C 2}=F_{1} \cdot \frac{D_{2}^{2}}{D_{1}^{2}} \cdot l_{C 2}$

310 Total external work $W_{\text {tot: }}$ :

$311 W_{t o t}=W_{\text {cond }}-W_{C 2}-W_{\exp }$

312 Due to the isothermal expansion, thermal energy equal to the expansion work has to be

313 added to the required thermal energy $E_{\mathrm{th}}$. which becomes:

$314 E_{t h}=A_{C 1} \cdot l \cdot(70 \cdot 4200+2260000) / 1.69+W_{\exp }+E_{\text {air }}$

315 With this input energy, the efficiency $\eta$ can be calculated:

$316 \eta=\frac{W_{t o t}}{E_{t h}}$

\section{Results and analysis}

319 Two test series were conducted, the first on 19.03.2014, and the second on 24.03.2104. Fig.

$3205 \mathrm{a}$ and $\mathrm{b}$ show the forces measured at the piston $\mathrm{P}$ for expansion ratios of $n=1$

321 (condensation only, no expansion) to $n=4$. The forces measured on 24.03.2014 (Fig. 5b)

322 are slightly smaller than those measured on 19.03., this was thought to be caused by

323 abrasion of the sealing rings, and subsequent increased air ingression. Fig. 5 indicates that

324 the tests are fairly repeatable. 


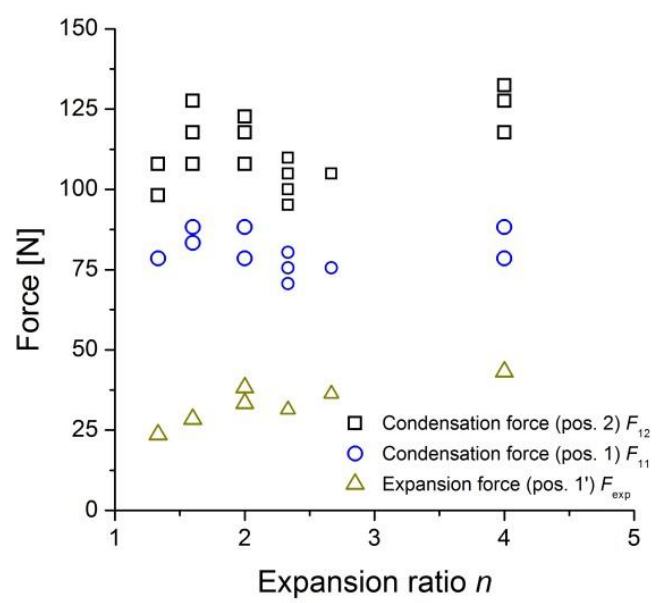

327

a. Tests 19.03.2014

Fig. 5: Measured forces

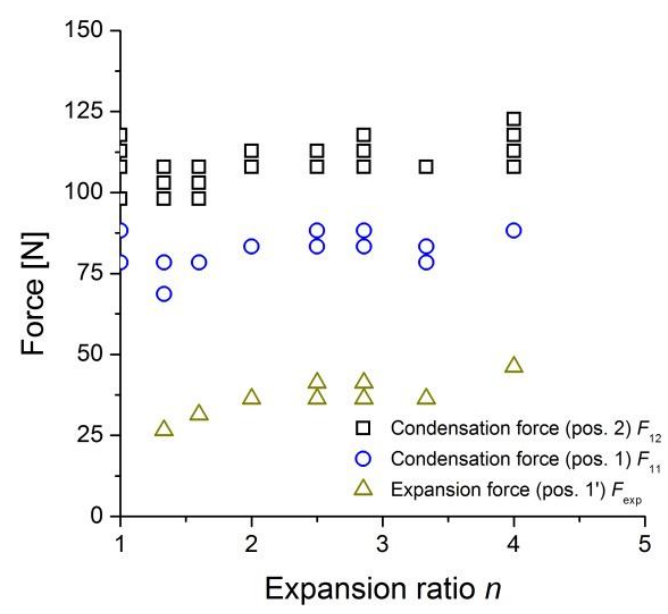

b. Tests 24.03 .2014

Table 1 shows the mechanical power and the thermal input power generated in the experiments. Column 2 shows the minimum and maximum power measured during the working stroke, column 3 the total mechanical output from Eq. 7 ( $n=1: 1)$ and Eq. 16 ( $n=2: 1$, $4: 1)$. In column 5 finally the thermal energy required is shown. It can be seen from columns 2 and 3 the work generated during the down stroke (working stroke) does not differ very much for the different expansion ratios. The total work (col. 3) for $n=1: 4$ is slightly less than the work from the tests with $n=1: 1$. However the thermal energy input for the expansion ratio $n$ $=4: 1$ is only a quarter of the energy input for the fully atmospheric cycle $(n=1: 1)$.

\begin{tabular}{|c|c|c|c|c|c|c|c|c|}
\hline 1 & \multicolumn{2}{|c|}{2} & \multicolumn{2}{|c|}{3} & \multicolumn{2}{|c|}{4} & 5 & 6 \\
\hline & $\begin{array}{r}W \\
\text { downst }\end{array}$ & $\begin{array}{l}\text { ok } \\
\text { oke [J] }\end{array}$ & Expans & $\begin{array}{l}\text { nork } \\
\text { ] }\end{array}$ & $\begin{array}{r}\text { Total } n \\
\text { stror }\end{array}$ & $\begin{array}{l}\text { ork per } \\
\text { e [J] }\end{array}$ & $\begin{array}{l}\text { Steam } \\
\text { vol. }\end{array}$ & $\begin{array}{l}\text { Thermal } \\
\text { work [J] }\end{array}$ \\
\hline $\begin{array}{l}\text { Expansion } \\
\text { ratio }\end{array}$ & Min. & Max & Min. & Max & Min. & Max. & & \\
\hline $1: 1$ & 17.7 & 21.6 & 0 & 0 & 14.0 & 17.1 & 346.8 & 523 \\
\hline $1: 2$ & 19.6 & 21.1 & 1.7 & 1.9 & 13.7 & 14.0 & 173.4 & 265 \\
\hline $1: 4$ & 19.6 & 22.1 & 3.2 & 3.2 & 12.8 & 13.9 & 86.7 & 136 \\
\hline
\end{tabular}

Table 1: Work measured during the experiments (Tests 19.03.2014)

Fig. 6 shows the efficiency calculated from the measurement values as a function of the expansion ratio $n$. The tests without expansion $(n=1)$ resulted in efficiencies of up to 0.032 . The average condensation pressure acting on the piston $P$ after condensation for all tests was $55 \mathrm{kPa}$. This means that the maximum theoretical conversion efficiency for a condensation stroke (pressure in $\mathrm{C} 1=45 \mathrm{kPa}$ abs.) is 0.036 , slightly higher than the experimental values. The highest measured efficiency for $n=4$ was 0.102 , exceeding even the theoretical maximum efficiency of the ideal atmospheric cycle $(0.065)$ by $60 \%$. 


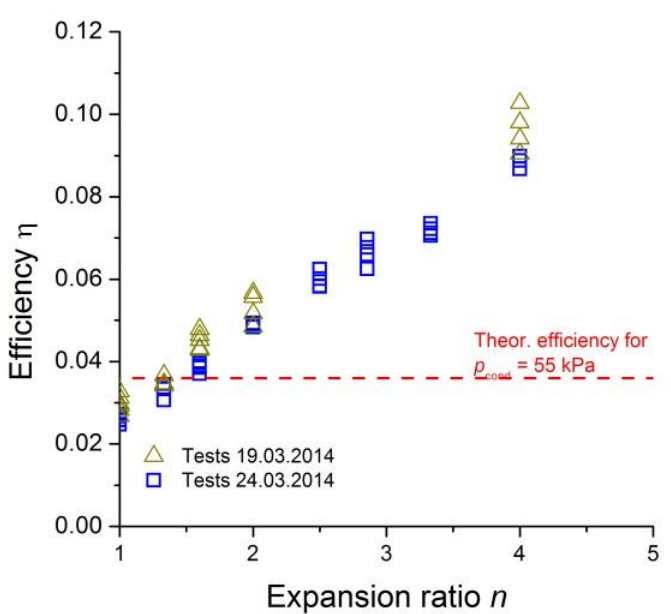

Fig. 6: Efficiencies

347 The working cycle was assumed to be isothermal, since the cylinder temperature was kept at $100^{\circ} \mathrm{C}$ by the hot water filled external jacket tube in which the cylinder was located. Internal temperatures were not measured, so that it is difficult to ascertain the actual degree of isothermal expansion. Theory as well as the low magnitude of the expansion work determined from the tests (only up to $16 \%$ of the work from the condensation stroke) indicate that temperature differences during expansion were small. This implies that near isothermal conditions prevailed.

The test rig was designed for single stroke operation. It may however be of interest to estimate what power output can be expected from a rotating engine. Assuming continuous operation, and a rotational speed of $120 \mathrm{rpm}$, the power output of the experimental rig would range from 24.6 to $30 \mathrm{~W}$ for a cylinder volume of $0.347 \mathrm{I}$. The engine's power density per unit swept volume can then be determined as $0.083 \mathrm{~kW} / \mathrm{l}$ cylinder volume. With improved sealing, a condensation pressure of $4 \mathrm{kPa}$ (abs.) should be possible. This would increase the efficiency to approximately $14 \%$, and the power density to $0.115 \mathrm{~kW} / \mathrm{l}$.

A real machine would however need to be significantly larger than the experimental rig. Also, a higher expansion ratio of $n=7$ to 10 would probably be chosen to increase engine efficiency. This comes however at the cost of power density. Assuming a cylinder diameter of $400 \mathrm{~mm}$, a stroke of $800 \mathrm{~mm}$, a speed of $90 \mathrm{rpm}$ and a twin cylinder machine for smoother running the power output for an expansion ratio of $n=1: 8$ would reach $13.7 \mathrm{~kW}$ for a thermal input of $96 \mathrm{~kJ}$. The machine would require a steam volume of $37.7 \mathrm{l} / \mathrm{s}$.

\section{Discussion}


The experiments conducted at Southampton University confirmed that the theoretically predicted concept of the atmospheric engine with forced expansion is feasible. The possibility to expand steam, and to condense the expanded steam, thereby increasing the efficiency of the atmospheric steam engine, was demonstrated.

For the evaluation of the experimental results presented in the previous section, the theoretical maximum efficiency of the ideal atmospheric engine (without any losses / with perfect vacuum) with $6.5 \%$ constitutes one benchmark. The residual pressure observed in the experiments after condensation was $45-46 \mathrm{kPa}$ (abs.), nowhere near a perfect vacuum. The maximum theoretical efficiency of the ideal atmospheric working stroke (without forced expansion) was therefore only $3.5 \%$. With maximum efficiencies of 8.9 to $10.2 \%$, this benchmark was exceeded by a factor of 2.8. Even the efficiency of the ideal atmospheric cycle was exceeded by $60 \%$.

The experiment suffered from an unsatisfactory sealing of the cylinder, which was caused by the use of a tube as cylinder. The brass tube had a deviation of the true diameter of $0.2 \%$ or approximately $1 \mathrm{~mm}$, which the O-ring seals could only partially compensate. In consequence, the minimum pressure in the cylinder at condensation did not drop below 45 $\mathrm{kPa}$ (abs.), limiting the possible work of the condensation stroke. During the expansion stroke only about $1 / 3$ of the theoretical pressure was reached, and a significant amount of air drawn into the cylinder.

\subsection{Performance}

The overall performance however was considered promising. The highest measured efficiency of $10.2 \%$ substantially exceeds values reported for much more complex ORC thermal engines for higher operating temperatures of $120^{\circ} \mathrm{C}(7.98 \%)$, [9]. A lower condensation pressure will be achievable with better sealing and a more accurate cylinder and piston. Condensation at $4 \mathrm{kPa}$ (abs.) should increase the efficiency from $10 \%$ to $15 \%$. In a real machine, there would be losses from the boiler and energy losses through the cylinder insulation, so that the actual efficiency from energy in to mechanical energy out would be somewhat lower.

For actual applications, the atmospheric engine does however have limitations:

1. The comparatively low energy density of unpressurized steam means that large volumes for cylinder and boiler are required.

2. The speed of the machine will also be low, it is currently estimated at $90 \mathrm{rpm}$ due to the long stroke. 
3. The condenser produces low-grade heat with temperatures approximately $10 \mathrm{~K}$ above ambient. The condenser fluid will need to be cooled down to ambient temperature, and the thermal energy will need to be released into the atmosphere.

The potential advantages can be listed as follows:

1. With boiler efficiencies of $90 \%$, and further $5 \%$ thermal losses in the cylinder, total system efficiencies $14 \%$ for $n=8$ seem achievable. The atmospheric engine with forced expansion therefore constitutes a significant improvement.

2. The ASE is simple compared e.g. with ORC engine systems, indicating cost effectiveness,

3. Operating temperatures are low compared with other thermal engines, widening the possible area of application.

4. The working fluid is cheap, readily available, non-toxic, not inflammable.

\subsection{Solar thermal system}

The work on cost-effective, medium temperature flat plate solar thermal collectors described in [6] indicates that for larger collectors (e.g. $3 \times 3 \mathrm{~m}$ area) are more efficient. For operating temperatures of $130^{\circ} \mathrm{C}\left(\mathrm{G}=800 \mathrm{~W} / \mathrm{m}^{2}\right)$, efficiencies of $60 \%$ are possible The overall mechanical efficiency (sun to shaft) of a collector combined with an atmospheric steam engine (operating temperature $T_{\mathrm{Ev}}=100^{\circ} \mathrm{C}$ ) can then be estimated as 9 to $9.5 \%$. This would probably give a sun-to-wire efficiency of approximately $8 \%$. It should be noted that the ORC engine reported in [9] had an engine-only efficiency of $7.98 \%$ for an operating temperature of $120^{\circ} \mathrm{C}$.

Overall efficiencies of the solar thermal system would be lower than those of e.g. PV systems. The atmospheric engine is however a simple machine, so that the overall costeffectiveness needs to be considered in the next development step.

\section{Conclusions}

Low and medium temperature thermal energy is available from many renewable energy. sources. The cost effective conversion of thermal into mechanical energy however still poses an engineering problem. One solution for thermal energy with temperatures of 100 to $150^{\circ} \mathrm{C}$ could be the atmospheric steam engine. Its theory was recently revisited in order to increase the machine's efficiency. The improved theory indicates that the addition of a forced expansion stroke can increase the theoretical efficiencies from 6.5 to $20 \%$. At Southampton University, a series of fundamental model tests was conducted in order to assess these predictions. The following conclusions were drawn. 
1. The theoretically postulated atmospheric cycle with forced expansion of steam is possible.

2. Air leakage through the seals limited the performance of the experimental machine.

3. The efficiency without expansion reached $3.2 \%$ with a condensation pressure of 46 $\mathrm{kPa}$ (abs.).

4. Efficiencies with forced expansion ranged from $4.1 \%$ for an expansion ratio of $1.33: 1$ to $10.2 \%$ for an expansion ratio of $4: 1$.

5. The theoretical maximum efficiency of the simple atmospheric cycle of $3.6 \%$ was exceeded by a factor of $2.8 \%$.

The concept of forced expansion was demonstrated successfully. A substantial increase in cycle efficiency was observed. The atmospheric engine with forced expansion has significant further development potential.

\section{References}

[1] EEA. 2014. European Environment Agency: Projected changes in effective solar radiation (http://www.eea.europa.eu/data-and-maps/figures/mean-changes-ineffective-solar, accessed 13.02.014).

[2] Kalogirou S.A., Solar thermal collectors and applications. Progr. in Energy and Combustion Science 30 (2004) 231-295.

[3] Tian Y. and Zhao C.Y. A review of solar collectors and thermal energy storage in solar thermal Applications. Applied Energy. 2013, 104. 538-553.

[4] AIT, 2008, Testbericht Gluatmugl HT 4.2m² (Test Report), available online: http://www.solid.at/images/stories/pdf/gluatmugl\%20ht\%20-\%20pruefbericht\%20\%20zuverlaessigkeitspruefung.pdf (accessed 16.03.2011).

[5] ISFH 2014. Entwicklung eines hocheffizienten Flachkollektors mit spektral selektiv beschichteter Zwischenverglasung (http://www.isfh.de/institut solarforschung/hocheffizienter-flachkollektor.php, accessed 01.03.2014).

[6] Prince J. Medium Temperature Solar Thermal Collector. Individual project. University of Southampton. 2013.

[7] Kongtragool B, Wongwises S. A four power-piston low-temperature differential Stirling engine using simulated solar energy as a heat source. Solar Energy 2008; 82: 493-500. 
472 [8] Quoilin S, Orosz M, Hemond H, Lemort V. Performance and design optimization of a low-cost solar organic Rankine cycle for remote power generation. Solar Energy 2011; 85: 955-966

[9] Li M, Wang J, He W, Gao L, Wang B, Ma S, Dai Y. Construction and preliminary test

[10] Quoilin S., Van Den Broek M., Declaye S. Dewallef P. and Lemort V. Technoeconomic survey of Organic Rankine Cycle (ORC) systems Renewable and Sustainable Energy Reviews 22 (2013) 168-186.

[11] Black NH, Davis HN. Practical physics for secondary schools. Fundamental principles and applications to daily life. London: Macmillan \& Comp.; 1913. (see also http://en.wikipedia.org/wiki/File:Newcomen6325.png)

[12] Thurston R H History of the Growth of the Steam engine. New York: D. Appleton \& Co; 1878 (available on http://en.wikipedia.org/wiki/File:Watt_steam_pumping_engine.JPG).

13] Dickinson HW. A short history of the steam engine. Cambridge: Cambridge University Press; 1938.

[14] Polytechn. Journ. Ueber Neuerungen an Kleindampfmaschinen. Polytechnisches Journal 1886; 259: 1-9 (http://dingler.culture.hu-berlin.de/article/pj259/ar259001, accessed 10.03.2014).

[15] Müller $G$, The atmospheric steam engine as energy converter for low and medium temperature thermal energy. Renewable Energy 2013; 53: 94-100.

[16] Dietzel F. and Wagner W. Technische Wärmelehre (Technical thermodynamics), $9^{\text {th }}$ 